\title{
INVARIANT SUBSPACES AND WEAKLY CLOSED ALGEBRAS
}

\author{
BY HEYDAR RADJAVI AND PETER ROSENTHAL
}

Communicated by P. R. Halmos, March 20, 1968

1. Introduction. One of the reasons that reducing subspaces of operators on a Hilbert space are more easily studied than invariant subspaces is the relation between reducing subspaces and von Neumann algebras. If $\mathfrak{A}$ is a von Neumann algebra containing the identity operator and $B$ is an operator such that every reducing subspace of $\mathfrak{A}$ reduces $B$, then $B$ is in $\mathfrak{A}$.

If $\mathfrak{A}$ is any algebra of operators and if $\mathfrak{A}^{\prime}$ is the algebra of all operators which leave invariant every invariant subspace of $\mathfrak{A}$, then clearly $\mathfrak{A} \subseteq \mathfrak{X}^{\prime}$. Professor P. R. Halmos has suggested the following definition.

Definition. An algebra $\mathfrak{U}$ of operators on a Hilbert space is reflexive if whenever every subspace invariant under $\mathfrak{A}$ is invariant under $B$, then $B$ is in $\mathfrak{A}$.

Examples of reflexive algebras are easily constructed: if $\mathfrak{F}$ is any family of subspaces of a Hilbert space $\mathfrak{S}$ and if $\mathfrak{A}$ is the algebra of all operators which leave every subspace in $\mathfrak{F}$ invariant, then $\mathfrak{A}$ is reflexive. Clearly, every reflexive algebra is weakly closed and contains the identity operator. Also every von Neumann algebra containing the identity is reflexive.

In this paper we consider two sufficient conditions that a weakly closed algebra be reflexive. Our work is based upon elegant techniques introduced in recent papers of D. E. Sarason [4] and W. B. Arveson [1]. The main results of Sarason and of Arveson may be stated as follows.

SARASON's THEOREM. If $\mathfrak{A}$ is a weakly closed algebra (containing the identity) of commuting normal operators, then $\mathfrak{A}$ is reflexive.

ARveson's Theorem. If $\mathfrak{A}$ is a weakly closed algebra which contains a maximal abelian von Neumann algebra and which has no invariant subspaces except $\{0\}$ and $\mathfrak{S}$, then $\mathfrak{A}$ is reflexive (i.e. $\mathfrak{A}$ is the algebra of all operators on $\mathfrak{E})$.

Below we give two theorems that are similar to the above. Our main result (Theorem 1) is a generalization of Arveson's Theorem.

\section{Statement of results.}

THEOREM 1. A weakly closed algebra which contains a maximal abelian von Neumann algebra and whose lattice of invariant subspaces is totally ordered is reflexive. 
Note that Arveson's Theorem is a special case of Theorem 1. Our proof of Theorem 1 shows that the hypothesis that the lattice be totally ordered can be slightly weakened.

COROLlaRy 1. Let $A$ be an operator whose lattice of invariant subspaces is totally ordered. Then there exists a Hermitian operator $H$ such that the weakly closed algebra generated by $A$ and $H$ is the algebra of all operators which leave invariant every invariant subspace of $A$.

A particular instance of Corollary 1 is the following.

Corollary 2. Let $V$ be the Volterra operator on $L^{2}(0,1)$, defined by $(V f)(x)=\int_{0}^{x} f(t) d t$. Let $H$ denote multiplication by the independent variable on $L^{2}(0,1)$, and for each $\alpha \in[0,1]$ let $M_{\alpha}=\left\{f \in L^{2}: f=0\right.$ a.e. on $[0, \alpha]\}$. Then the weakly closed algebra generated by $V$ and $H$ is

$$
\left\{A: A M_{\alpha} \subseteq M_{\alpha} \text { for all } \alpha\right\} \text {. }
$$

THEOREM 2. If $\mathfrak{H}_{0}$ is a weakly closed algebra containing the identity and if $\mathfrak{A}=\mathfrak{A}_{0} \otimes I$, where $I$ is the identity operator on an infinite-dimensional space, then $\mathfrak{A}$ is reflexive.

The hypotheses of Theorem 2 state that $\mathfrak{A}$ can be regarded as an algebra of "inflations" in the sense defined by Halmos [2]. Theorem 2 is of in terest primarily because the algebra $\mathfrak{A}_{0}$ is completely arbitrary, and hence $\mathfrak{A}$ need not contain any nice (i.e. normal, shift, etc.) operators.

3. Remarks. In the course of proving Theorem 1, we give a simplified proof of Arveson's Theorem. The corollaries follow easily from Theorem 1. The proof of Theorem 2 is very easy using ideas of Sarason's. We shall publish proofs of Theorems 1 and 2 and some other results elsewhere.

It seems to us that the problem of finding other sufficient conditions that algebras be reflexive is interesting. It is also interesting to seek properties of reflexive algebras (see [3] for some results related to this).

\section{REFERENCES}

1. W. B. Arveson, $A$ density theorem for operator algebras, Duke Math. J. 34 (1967), 635-647.

2. P. R. Halmos, Shifts on Hilbert spaces, J. Reine Angew. Math. 208 (1961), $102-112$.

3. R. V. Kadison and I. Singer, Triangular operator algebras, Amer. J. Math. 82 (1960), 227-259.

4. D. E. Sarason, Invariant subspaces and unstarred operator algebras, Pacific J, Math. 17 (1966), 511-517.

UNIVERSITY OF TORONTO 